\title{
Distance Dependence of Path Loss for Millimeter Wave Inter-Vehicle Communications
}

\author{
Satoshi TAKAHASHI, Akihito KATO, Katsuyoshi SATO, Masayuki FUJISE \\ National Institute of Information and Communications Technology, 3-4 Hikarino-oka, Yokosuka 239-0847, Japan
}

\begin{abstract}
Millimeter-wave path loss between two cars was measured to obtain the general applicable distance for inter-vehicle communication systems in real environments. An abrupt and substantial increase in path loss due to interruption, curves, and different-lane traveling has been a major concern in inter-vehicle communications. The path loss measurements were carried out using $60-\mathrm{GHz} C W$ radiowaves and standard horn antennas on metropolitan highways and regular roads. Because the propagation loss is traffic-dependent, the highways were classified into uncrowded and crowded highways, and the regular roads were classified into uncrowded and crowded roads. The path loss for the highways exhibited 2nd-power-law attenuation and that for the regular roads exhibited 1stpower-law attenuation with an increase in inter-vehicle distance. Additional losses of $15 \mathrm{~dB}$ for the highways and 5 $d B$ for the regular roads were observed when the intervehicle distance was more than approximately $30 \mathrm{~m}$. Thus, we were able to demonstrate millimeter-wave inter-vehicle communications at an inter-vehicle distance of more than $100 \mathrm{~m}$.
\end{abstract}

\section{Keywords}

Millimeter-wave inter-vehicle communications, distance characteristics of path loss, ITS.

\section{Introduction}

Millimeter-wave inter-vehicle communications can potentially be used in intelligent transport systems (ITS) of the future, which will be equipped with brake assistance, driving information transmission, and safe driving assistance system [1]. Several studies on inter-vehicle networks and radio propagation in millimeter-wave bands have been conducted.

The inter-vehicle communication networks in these studies were based on radio propagation models. The initial connection time in systems employing R(reservation)ALOHA was evaluated assuming a two-path model of direct and ground reflection radiowave propagation [2], and the probability of successful transmission in a multihop ALOHA communication system was calculated [3]. The use of a collision sensed multiple access (CSMA) method for inter-vehicle communications was examined, and the appropriate number of bits assigned for the packet was obtained [4]. Because these studies use radio propagation models, it is important to know radio propagation in inter-vehicle communications. A number of radio propagation models rather more realistic have been proposed and the characteristics of path-loss variation have been derived. A time-variant simulation has been conducted to evaluate the distance dependence of path loss [5]. An inter-vehicle distance has been obtained from the mean inter-vehicle distance for highway, suburban, or forest environments. The distance dependence of path loss has been calculated by ray tracing assuming a generic environment. Fading due to vertical fluctuation has been reproduced assuming a two-path model using a car suspension model of the freedom of one [6]. Empirical measurements of radio propagation are also important because the path loss in inter-vehicle communications is site-dependent. The transmission performance of ASK, FSK, PSK, and QAM, as well as path loss, has been measured to estimate the space diversity gain [7]. The results for highways showed that the path loss in a quasi static environment can be accounted for by interference between two dominant radiowaves. The results also suggested that the path loss in the Raleigh amplitude distribution was caused by the vertical fluctuation of the cars. Nevertheless an abrupt and substantial increase in path loss due to interruption, curves, and different-lane traveling has been a major concern in inter-vehicle communications.

We derived the distance dependence of path loss to determine the general applicable distance between cars for inter-vehicle communication systems in real environments. The distance dependence of path loss was measured for the following for environments: uncrowded and crowded highways, and uncrowded and crowded regular roads. Based on the results we determined the applicable distance between cars for store-and-forward multimedia data transmission and brake assistance.

\section{Path-Loss Measurements}

Although the delay characteristics are generally important in radio transmission, it is the path loss that determines the performance of radio transmission in millimeter-wave inter-vehicle communications. This is because the delay spread and the maximum excess delay 
time are significantly smaller than the symbol duration, and can be less than 1/20 of the ratio: for example, in binary transmission at a rate of $100 \mathrm{Mbit} / \mathrm{s}$, the symbol duration is $10 \mathrm{~ns}$. The dominant paths in inter-vehicle communications are thought to be the direct and ground-reflected paths [7], and the difference in the lengths of these paths is $9.0 \mathrm{~mm}$ when the inter-vehicle distance is $20 \mathrm{~m}$ and both the transmission and reception antennas are $0.3 \mathrm{~m}$ high (the difference is $1.8 \mathrm{~mm}$ when the distance is $100 \mathrm{~m}$ ). The arrival-time differences are only 0.03 and $0.006 \mathrm{~ns}$, and the ratio of the symbol duration to the maximum excess delay time is less than 0.003. Therefore the delay characteristics do not significantly affect the radio transmission.

The statistical modeling of path loss is preferable to the site-specific modeling such as two-path model because radio transmission is site-dependent, and both sides of communication run independently. The path loss may vary significantly due to obstruction, curves, reflection of surrounding cars, and antenna directivity resulting from the traveling lane.

There are two methods of path-loss measurement. One focuses on measuring the initial connection, and the other focuses on connection maintenance. Authors in Ref. [7] focused on the initial connection, measured the path loss at a constant inter-vehicle distance and constant velocity for cars traveling in the same lane, and determined the amplitude distribution and fade duration resulting from obstruction and reflection of the ground and surrounding objects. In this paper, we focus on connection maintenance of inter-vehicle communications. We measured the path loss for two cars traveling in either same or different lanes.

Measurements were performed on highways and regular roads. The highways were the Yokohama-Yokosuka Highway and the Hodogaya Bypass; these highways link western Tokyo and Yokosuka city, and they are about $40 \mathrm{~km}$ long in total. The highways are mostly 4-lane highways but they also have 2- and 6-lane segments. The regular roads were several roads in Yokosuka city, and almost all were 2-lane roads. The measurements were performed at a continuous-wave (CW) frequency of $60 \mathrm{GHz}$ and a transmission power of $10 \mathrm{~mW}$. Vertically-polarized standard horn antennas with a gain of $23 \mathrm{dBi}$ were used, and they were attached to the transmission and reception cars. The maximum measurable path loss and the dynamic range are approximately $140 \mathrm{~dB}$ and $60 \mathrm{~dB}$. A simple sketch of the measurement geometry is illustrated in Fig. 1. Each car was equipped with an industrial GPS receiver, and the instant location of each car associated with each time was recorded on a personal computer. In the reception car, successive analog reception power was recorded on a digital audio tape (DAT) recorder having a GPS-based timeassociation function. To reflect the effect of the drivers' intentions on the path loss, we used professional drivers who were not told the goal of the experiment. Therefore the path loss was caused not only by obstruction, ground reflection, and surrounding object reflection, but also by

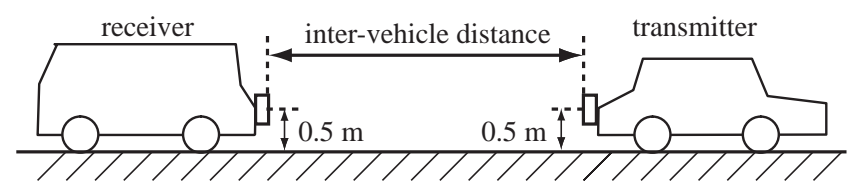

Fig. 1. A sketch of the measurement geometry.

antenna directivity resulting from the cars traveling in different lanes and the drivers' intentions such as give-away and passing other cars. Each car was also equipped with a video camera and a GPS-based video cassette recorder (VCR) to record the surrounding environment while measurements.

After these measurements, we obtained a curve that relates between DAT-recorded values and reception power by providing known signal powers to the receiver. Then we obtained the time-variant path loss using the curve, and estimated the distance dependence of the path loss using the instant locations of the cars.

\section{Distance Dependence of Path Loss}

The distance dependence of the path loss varied with the surrounding environment. Therefore we classified the environment into two types, uncrowded and crowded. The word "uncrowded" here refers to an environment in which the two cars could run freely, occasionally passing other cars. The word "crowded” refers to traffic jams, when the cars could not move and the drivers could not change lanes. The classification was made based on visual observation of road conditions. Although these qualitative descriptions are not precise, they may fit for most of general road conditions. The instant path loss in the uncrowded highway is shown in Fig. 2. The instant path losses varied more than $40 \mathrm{~dB}$ at the same inter-vehicle distance. The 5th-, 50th-, 95th-, and 99th-percentile path losses at an interval of $2.5 \mathrm{~m}$ of the back-and-forth areas were calculated every $5 \mathrm{~m}$. These values are also shown in Fig. 2 except for the 99th-percentile path loss. The percen-

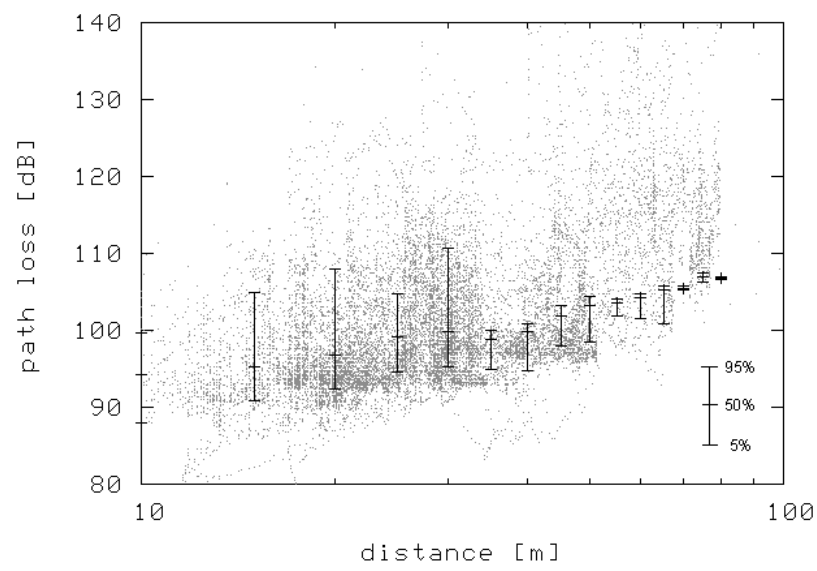

Fig. 2. Instant path losses and the percentile path losses for the uncrowded highways. 


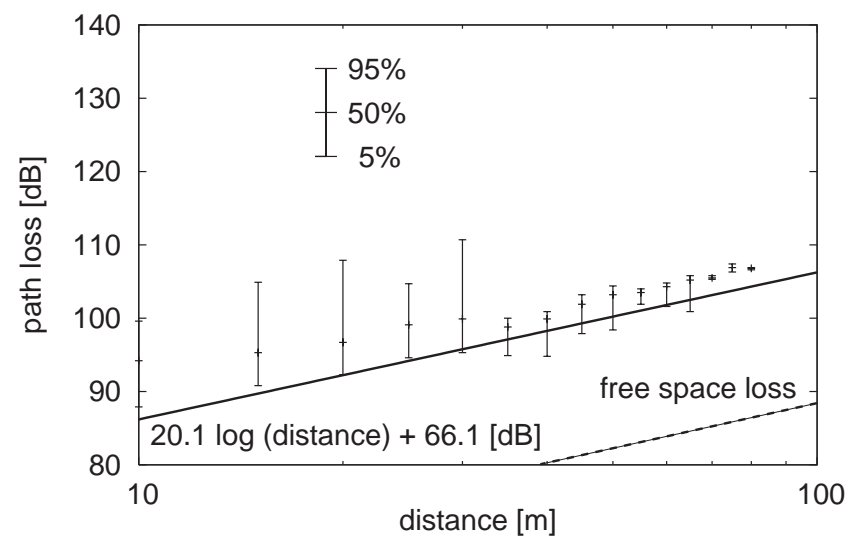

Fig. 3. Distance dependence of path losses for the uncrowded highways.

tile path losses at a distance of more than $80 \mathrm{~m}$ are not shown in the figure because few samples were collected. When we define the $90 \%$ confidence interval for the path losses as the difference between the 5th and the 95thpercentile path losses, we can see that the instant path losses exceeding the 95th-percentile path loss are widely distributed but those smaller than the 5th-percentile path loss tend to concentrated on the percentile path loss. The minimal reception power falls dramatically.

The distance dependence of the $90 \%$ confidence interval for the path losses extracted from Fig. 2 is shown in Fig. 3. The regression line for the 5th-percentile path losses is also shown. The 5th-percentile path loss decreases with an increase of the inter-vehicle distance. The regression line followed the 2nd-power-law attenuation (the reception power decreased at 2nd power exponent with an increase in the distance), which is similar to free-space attenuation. The excess loss defined as the difference between the path loss and the free-space path loss derived from the Friis' law, was about $15 \mathrm{~dB}$. The 5th-percentile path losses could be regressed well but the 95th-percentile path losses varied. It is possible that the measurement was done when the two cars were traveling side by side which significantly increases the path loss because of the antenna directivity. In this situation, the path loss may vary with a slight change in the environment. For example in Fig. 3, the data at an inter-vehicle distance ranging from 10 to $30 \mathrm{~m}$ are not suitable for the 95th-percentile path loss regression.

The distance dependence of the 5th-percentile path losses for the crowded highways is shown in Fig. 4. The 5th-percentile path losses followed 2nd-power-law attenuation, just like the path losses for the crowded highways did. When the inter-vehicle distance was more than $35 \mathrm{~m}$, an additional path loss of $15 \mathrm{~dB}$ was observed. This may have resulted from line-of-sight obstruction. The distance of 35 m may be a threshold that cars are obstructed on highways due to the drivers' intensions. The 5th-percentile path losses for an inter-vehicle distance of more than $35 \mathrm{~m}$ also followed 2nd-power-law attenuation.

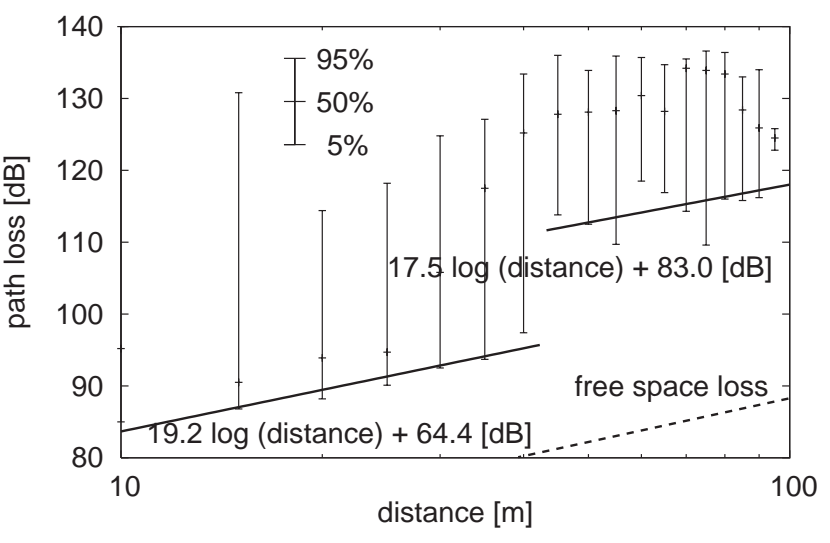

Fig. 4. Distance dependence of path losses for the crowded highways.

The path-loss variation is defined as the range of the $90 \%$ confidence interval. The variations in instant path losses for the uncrowded and crowded highways are compared in Fig. 5. In general, the heavier the traffic, the more the variation. When the inter-vehicle distance was $20 \mathrm{~m}$, the variation for the uncrowded highways was $15 \mathrm{~dB}$, but the variation for the crowded highways was $26 \mathrm{~dB}$. For the crowded highways and an inter-vehicle distance of $10 \mathrm{~m}$, the path loss reached $56 \mathrm{~dB}$, the highest value for all the environments. This may have been caused by both of the cases that the two cars were side by side and in line while the measurement. At an inter-vehicle distance between 20 and $80 \mathrm{~m}$, the difference in the variation between the uncrowded and crowded highways was 11-30 dB. With an increase in the traffic, the path-loss variation also increased by approximately $20 \mathrm{~dB}$. The variation decreased with an increase in the inter-vehicle distance.

The cumulative distribution functions (CDFs) of the path losses for the highways are compared in Fig. 6.

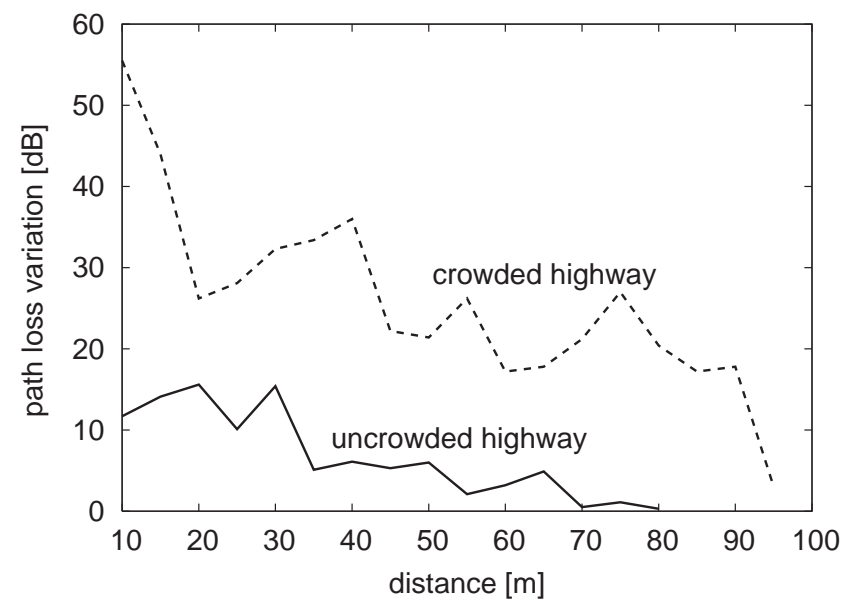

Fig. 5. Distance dependence of path-loss variation for the highways. 


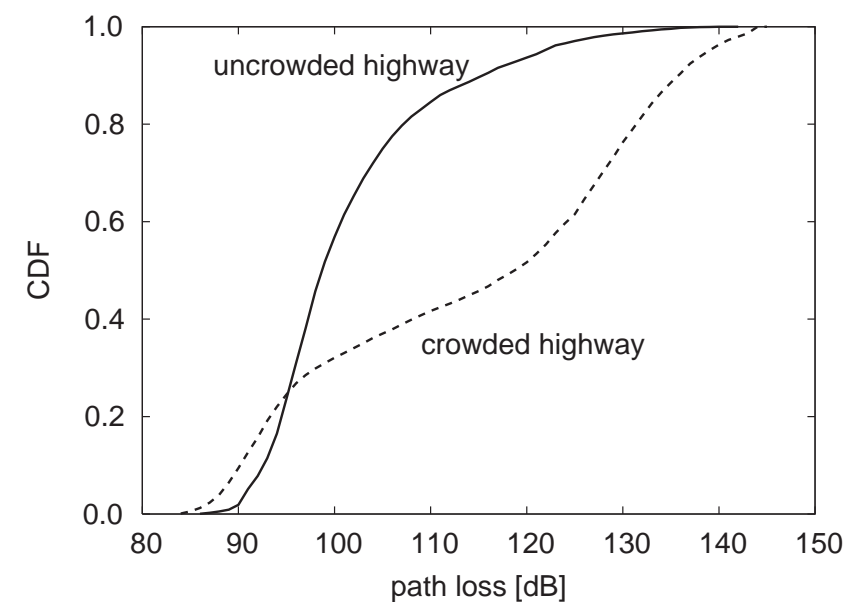

Fig. 6. CDFs of path losses for uncrowded and crowded highways.

The CDF of path losses for the uncrowded highways can be expressed as a single probability function. In contrast, the CDF for the crowded highways consists of distributions of path losses ranging from 85 to $95 \mathrm{~dB}$ and from 125 and $140 \mathrm{~dB}$. For the crowded highways, the cars experienced both of the low and high path-loss cases while the measurement.

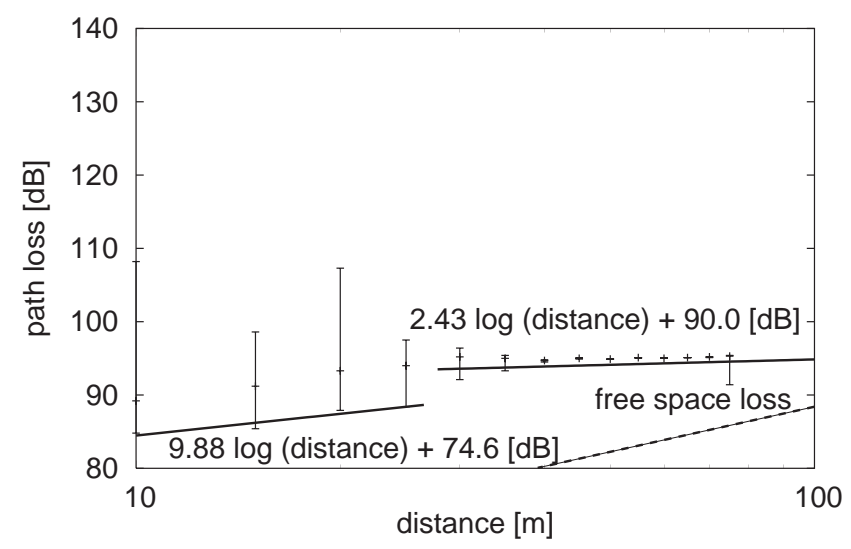

Fig. 7. Distance dependence of path losses for the uncrowded regular roads.

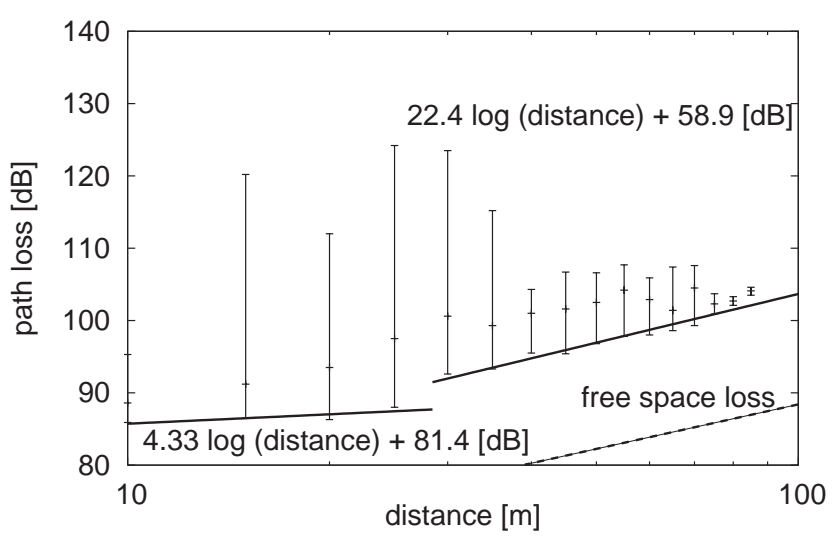

Fig. 8. Distance dependence of path losses for the crowded regular roads.
We also obtained the confidence interval for the path losses and their variation for the regular roads. The distance dependence of the path losses for the uncrowded and crowded regular roads is shown in Figs. 7 and 8. In Fig. 7, the 5th-percentile path losses for the uncrowded regular roads at an inter-vehicle distance between 10 and $25 \mathrm{~m}$ obey 1st-power-law attenuation, and those at a distance of more than $25 \mathrm{~m}$ follow below 1st-power-law attenuation. Confidence intervals for the path losses at a distance of more than $75 \mathrm{~m}$ are not plotted because few samples were collected. In general, the path losses for the regular roads were lower than for the highways. This may be because the possibility of cars traveling in different lanes as well as interruption is low on regular roads. The path-loss variation for the uncrowded regular roads at an inter-vehicle distance between 30 and $70 \mathrm{~m}$ was less than a few decibels. The uncrowded regular roads had the lowest path loss and the lowest path-loss variation among all the environments. The distance dependence of the 5th-percentile path losses for the crowded regular roads shown in Fig. 8 follows below 1st-power-law attenuation at an inter-vehicle distance between 10 and $25 \mathrm{~m}$, and it follows 2nd-power-law attenuation at a distance of more than $30 \mathrm{~m}$. Excess path losses that might have been caused by cars interruption were observed on both types of regular roads. These excess path losses, however, were approximately $5 \mathrm{~dB}$ and were smaller than those observed on the highways. In general, the possibility of interruption and the resulting path loss on regular roads is smaller than on highways.

The path-loss variations for the uncrowded and crowded roads are compared in Fig. 9. As can be seen in the figure, the path-loss variation decreases with an increase in the inter-vehicle distance, and the path-loss variation for the crowded roads is higher than for the uncrowded roads, as is the case for the highways. The increase in the path-loss variation due to heavier traffic on the crowded roads was approximately $10 \mathrm{~dB}$. The path-loss variation for the regular roads was $10 \mathrm{~dB}$ smaller than for the highways.

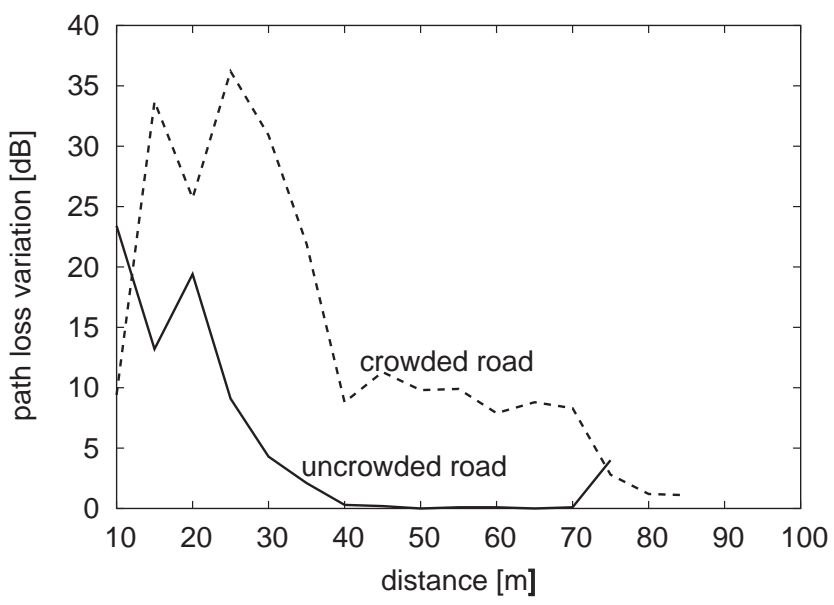

Fig. 9. Path-loss variations for the uncrowded and crowded roads. 


\begin{tabular}{|c|c|c|}
\hline roads & $\begin{array}{c}\text { 5th-percentile } \\
\text { path loss [dB] } \\
(d: \text { distance }[\mathrm{m}])\end{array}$ & $\begin{array}{c}\text { applicable } \\
\text { distance } \\
\text { range [m] }\end{array}$ \\
\hline uncrowded highway & $20.1 \log _{10} d+66.1$ & $10-100$ \\
\hline crowded highway & $19.2 \log _{10} d+64.4$ & $10-40$ \\
& $17.5 \log _{10} d+83.0$ & $45-95$ \\
\hline uncrowded regular & $9.88 \log _{10} d+74.6$ & $10-25$ \\
road & $2.43 \log _{10} d+90.0$ & $30-75$ \\
\hline crowded regular & $4.33 \log _{10} d+81.4$ & $10-25$ \\
road & $22.4 \log _{10} d+58.9$ & $30-85$ \\
\hline
\end{tabular}

Tab. 1. Equations for calculating the 5th-percentile path losses for various roads.

\begin{tabular}{|c|c|c|}
\hline roads & $\begin{array}{c}\text { 99th-percentile } \\
\text { path loss [dB] } \\
(d \text { : distance [m] })\end{array}$ & $\begin{array}{c}\text { applicable } \\
\text { distance } \\
\text { range [m] }\end{array}$ \\
\hline uncrowded highway & $19.4 \log _{10} d+70.6$ & $35-80$ \\
\hline $\begin{array}{c}\text { crowded highway } \\
10.2 \log _{10} d+111.7\end{array}$ & $\begin{array}{c}20-35 \\
40-80\end{array}$ \\
\hline $\begin{array}{c}\text { uncrowded regular } \\
\text { road }\end{array}$ & $1.60 \log _{10} d+125.9$ & $40-75$ \\
\hline $\begin{array}{c}\text { crowded regular } \\
\text { road }\end{array}$ & $8.95 \log _{10} d+91.3$ & $40-70$ \\
\hline
\end{tabular}

Tab. 2. Equations for calculating the 99th-percentile path losses for various roads.

Tab. 1 and Tab. 2 show the equations for calculating the 5th- and 99th-percentile path losses for various roads in order to obtain the distance between cars for inter-vehicle communications applications. The directivity of the antennas has the key influence on the results. Yet the results may virtually hold for most of millimeter wave inter-vehicle communication systems because a few configurations of the millimeter-wave inter-vehicle communication antennas result in the counterpart directivity.

\section{Distance between Cars for Inter-Ve- hicle Communications Applications}

\subsection{Store-and-Forward Multimedia Data Transmission}

One possible application of millimeter-wave intervehicle communications is for store-and-forward multimedia data transmission. In this case, the system transmits traffic information and neighborhood-related data from one car to another by using a flooding (sequential retransmission) method. We assume that only $5 \%$ of low path-loss time can be used for inter-vehicle data transmission because not all cars will have the system.

A transmission power of $10 \mathrm{dBm}$, a transmission and reception antenna gain of $23 \mathrm{dBi}$, and a minimum reception power of $-70 \mathrm{dBm}$ are assumed here. These assumptions lead to a maximum allowable propagation loss of $126 \mathrm{~dB}$. Substituting the maximum allowable loss into the 5th-percentile path-loss equations shown in Tab. 1, we find that the distance for inter-vehicle communications can exceed $100 \mathrm{~m}$ in all the environments we have studied. The key to improve the store-and-forward multimedia transmission is the medium access method utilizing a $5 \%$ of low path-loss time.

\subsection{Brake Assistance}

Another application of inter-vehicle communications is for brake assistance, which the communication system receives brake information from the front car and assists the braking procedure.

The system requires that there be an always-on channel. A 99\% availability is assumed as the approximation of the always-on channel because the availability decreases with interruption. A maximum allowable loss of $126 \mathrm{~dB}$ is also assumed for break assistance application. According to Tab. 2, the applicable distance in this case can exceeds $100 \mathrm{~m}$ for uncrowded highways and both uncrowded and crowded regular roads. However, on crowded highways, the large path-loss variation shortens the applicable distance to $27 \mathrm{~m}$. This large path-loss variation is a problem in using millimeter-wave inter-vehicle communications for brake assistance.

\section{Conclusion}

Millimeter-wave path loss between two cars was measured to determine the general applicable distance for inter-vehicle communication systems in real environments. The path loss, rather than the delay characteristics, determines the performance of radio transmission in millimeterwave inter-vehicle communications. In our path-loss measurements, we focused on the drivers' intentions such as give-a-way and passing other cars as well as the surrounding-object effects such as curves.

The measurements were carried out on highways and regular roads. The environments were classified into uncrowded and crowded environments based on visual observation. The word "uncrowded" was used to describe environments where the cars could run freely, occasionally passing other cars. The word "crowded" was used to describe traffic jams, where the cars could not move at all or when the drivers could not change lanes. The 5th-, 50th-, 95th-, and 99th-percentile path losses were obtained as representative path losses. For the highways, the distance dependence of the 5th-percentile path losses followed 2ndpower-law attenuation with an increase in the inter-vehicle distance. In contrast, the distance dependence of 5thpercentile path losses for the regular roads followed below 1st-power-law attenuation. An excess path loss of about 15 $\mathrm{dB}$ was observed on the crowded highways and an excess 
path loss of about $5 \mathrm{~dB}$ was observed on regular roads at an inter-vehicle distance of more than $30 \mathrm{~m}$. The path-loss variation increased when the inter-vehicle distance decreased or when the traffic increased.

Using the distance dependence of path loss, we estimated the distance for store-and-forward multimedia data transmission applications and the brake assistance application. The applicable distance for multimedia data transmission using a 5\% low path-loss time was more than $100 \mathrm{~m}$ for all the observed environments. The key to implementing multimedia transmission is the medium access method. For brake assistance assuming a 99\% availability of transmission, the applicable distance for the uncrowded highways and both uncrowded and crowded regular roads was more than $100 \mathrm{~m}$, but this distance was limited to $27 \mathrm{~m}$ for the crowded highways because of the large path-loss variation. Large path-loss variation is a problem in millimeter-wave inter-vehicle communications.

\section{Acknowledgements}

The authors would like to thank the anonymous reviewers for their comments and suggestions.

\section{References}

[1] IIDA, T. Wireless communications R\&D in the science and technology policy in Japan. IEICE Trans. Electron. March 2002, vol. E85-C, no. 3, pp. 419-427.

[2] VERDONE, R. Performance evaluation of R-ALOHA for intervehicle communications at millimeter waves. In IEEE International Symposium on Personal, Indoor and Mobile Radio Communications (PIMRC '96). Oct. 1996, vol. 2, pp. 658-662.

[3] VERDONE, R. Multihop R-ALOHA for intervehicle communications at millimeter waves. IEEE Trans. on Vehicul. Technol., Nov. 1997, vol. 46, no. 4, pp. 992-1005.

[4] OHYAMA, T., NAKABAYASHI, S., SHIRAKI, Y., TOKUDA, K. A study of real-time and autonomous decentralized DSRC system for inter-vehicle communications. In IEEE International Conference on Intelligent Transportation Systems, 2000, pp. 190-195.

[5] MAURER, J., SCHAFER, T. M., WIESBECK, W. A realistic description of the environment for inter-vehicle wave propagation modelling. In IEEE Vehicular Technology Conference Fall (VTC 2001-Fall), 2001, vol. 3, pp. 1437-1441.

[6] MIZUTANI, K., KOHNO, R. Analysis of multipath fading due to two-ray fading and vertical fluctuation of the vehicles in ITS intervehicle communications. In IEEE International Conference on Intelligent Transportation Systems, 2002, pp. 318-323.

[7] KATO, A., SATO, K., FUJISE, M., KAWAKAMI, S. Propagation characteristics of $60-\mathrm{GHz}$ millimeter waves for ITS inter-vehicle communications. IEICE Trans. Commun., Sept. 2001, vol. E84-B, no. 9, pp. 2530-2539.

\section{About Authors...}

Satoshi TAKAHASHI received B.E., M.E., and Ph.D. degrees from Tokyo Denki University, Japan, in 1990,
1992, and 2001. In 1992 he joined Hitachi, Ltd., where he engaged in research on indoor radio propagation and the ray tracing prediction, and in the development of radio communication systems. From 1996 to 1999, he was a research engineer in YRP Key Tech Labs, where he engaged in research on microwave mobile radio propagation and the transmission performance prediction for future high data rate radio mobile communication systems. Since 2002 he is with National Institute of Information and Communications Technology (NICT), where he engages in research on radio propagation and MIMO transmission. Dr. Takahashi is a member of IEICE, IEEE, SITA (Society of Information Theory and its Applications), and IPSJ (Information Processing Society of Japan).

Akihito KATO was born in Osaka, Japan in 1965. He received the B.E. degree of electrical engineering from Doshisha University, Japan in 1989 and he also received D.E. degree of electrical engineering from Doshisha University in 1994. He joined National Institute of Information and Communications Technology (NICT) in 1994. Since then, he has been engaged in research on millimeter-wave indoor propagation and millimeter-wave wireless communication systems such as ultra high-speed wireless LAN system or inter-vehicle communication system on ITS. Dr. Kato is a member of IEICE and IEEE.

Katsuyoshi SATO was born in Iwate, Japan in 1967. He received the B.S. and M.S. degrees in electrical engineering from Tohoku University, Sendai, Japan, in 1989 and 1991, respectively. In 1991, he joined National Institute of Information and Communications Technology (NICT). He has been engaged in research on radio propagation characteristics of millimeter-wave, such as indoor radio propagation and millimeter-wave remote sensing. His current research interests include wireless technologies for ITS. He is a member of IEICE and JPS (Physical Society of Japan).

Masayuki FUJISE was born in Fukuoka, Japan in 1950. He received the B.S., M.S. and Dr.Eng.degrees, in communication engineering from Kyushu University, Fukuoka, Japan, in 1973, 1975 and 1987, respectively and the M.Eng.degree in electrical engineering from Cornell University, Ithaca, NY, in 1980. He joined KDD in 1975 and was with the R\&D Laboratories being engaged in research on optical fiber measurement technologies for optical fiber transmission systems. In 1990, he joined ATR Optical and Radio Communications Research Laboratories, where he managed research on optical intersatellite communications and active array antenna for mobile satellite communications. Since he joined National Institute of Information and Communications Technology (NICT) in 1997, he has been an executive manager of millimeterwave applications group. He is now interested in radio on fiber technology and software radio technology. Dr. Fujise is the recipient of the Jack Spergel Memorial Award of the 33rd International Wire \& Cable Symposium in 1984 and he is a member of IEEE. 